Año 26 No. 94

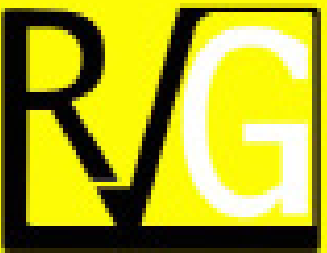

Abril-Junio, 2021

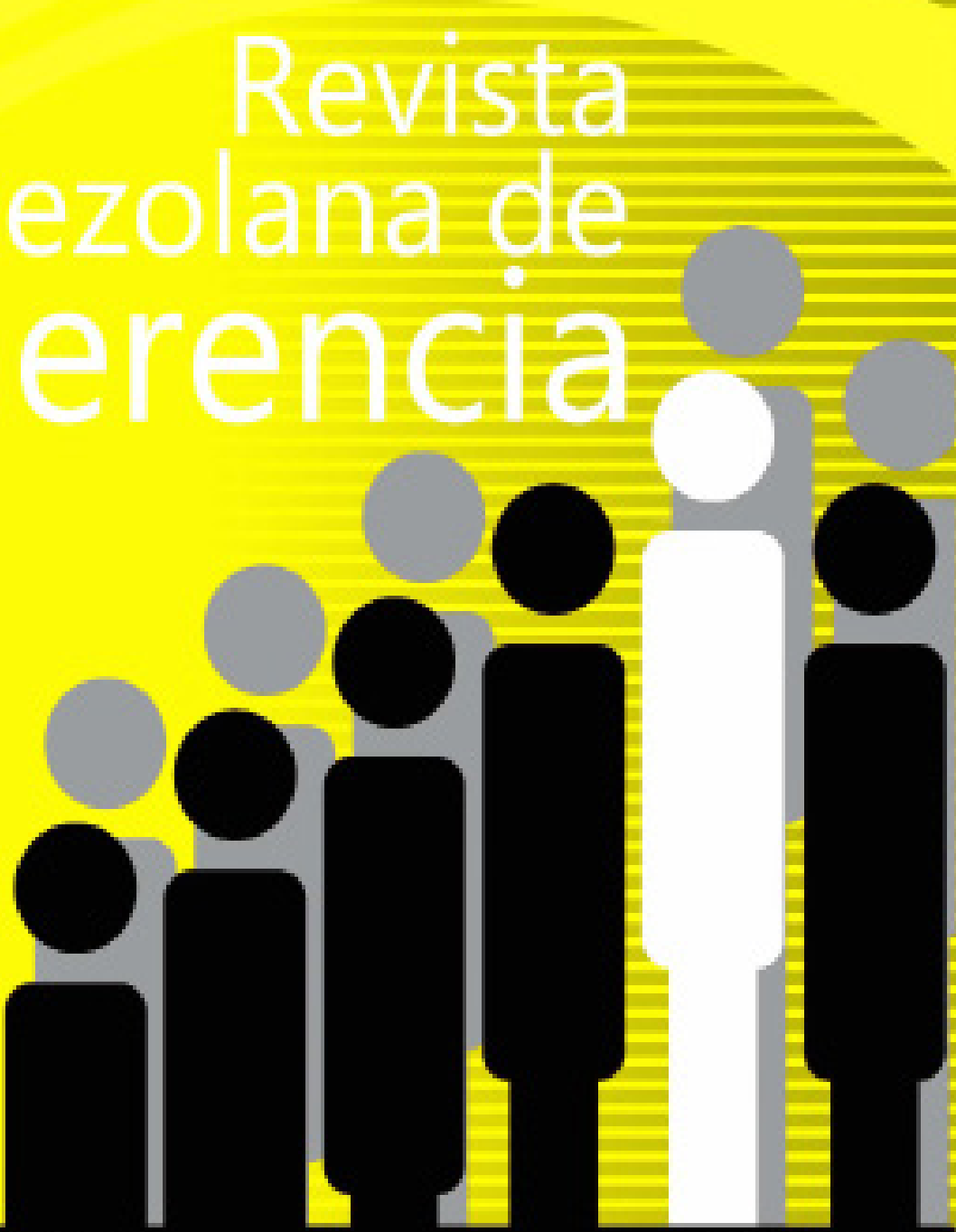

UNMERSDAD DEL ZULIA ILUZ) Fatultad de Cienthas Eennamicts y Baciales 


\title{
Gestión de riesgo de desastres en el marco de la cultura preventiva
}

\author{
Barra Tello, Tamara Carolina* \\ Salvatierra Melgar, Angel ${ }^{* \star}$ \\ Candia Haro, Isaac Moisés ${ }^{* *}$ \\ Vargas-Vargas, Gautama ${ }^{* \star *}$
}

\section{Resumen}

Enfrentarse al problema ambiental que vive el planeta, es uno de los retos más grandes al garantizar la permanencia del ser humano, según lo expresado en la Conferencia Científica de las Naciones Unidas, también conocida como la Primera Cumbre para la Tierra; la gestión de riesgos estratégicamente planificada y aplicada, allana el camino para obtener una ventaja competitiva y una ventaja decisiva para las organizaciones públicas. El objetivo de la investigación fue analizar la gestión de riesgo de desastres en el macro de la cultura preventiva desde las experiencias vividas de los trabajadores de un hospital público de Lima. El presente estudio se enmarca en el paradigma interpretativo bajo un enfoque cualitativo, en cuanto al diseño de investigación, Hernández \& Mendoza (2018) lo enmarcan dentro del diseño fenomenológico interpretativo al describir, explorar, pero sobre todo al comprender el contexto en el cual se desarrolla la investigación debido a la flexibilidad del proceso que se va adaptando a cada realidad, la unidad de análisis fueron 10 colaboradores de la brigada del hospital. Los resultados indican que la cultura preventiva incide de forma positiva en los trabajadores del hospital público; impactando la prevención positivamente no solo en la limitación de la gravedad de los daños que produzca el desastre, sino también en la capacidad de recuperación que tendrá el

Recibido: 20.10.20 Aceptado: 15.02.21

* Maestra en gestión de servicios de salud, enfermera de profesión, con especialidad en emergencias y desastres del hospital Dos de Mayo. E-mail: tbarra@ucvvirtual.edu.pe ORCID: https://orcid.org/0000-0002-5352$\underline{2887}$

** Docente de la universidad Nacional Mayor de San Marcos, doctorado en ciencias de la educación, ex coordinador de proyectos y desarrollo, E-mail: ansalme2@gmail.com ORCID: https://orcid.org/0000-0003-2817$\underline{630 X}$

*** Magíster en Gestión Pública y Gobernabilidad de la Universidad César Vallejo, especialista en políticas públicas y gobernabilidad. Trabajo como Coronel de la Policía Nacional del Perú, Lima - Perú, E-mail: isaaccandia@gmail.com ORCID: https://orcid.org/0000-0002-3927-4848

**** Magíster en Tecnología Educativa de la Universidad César Vallejo. Docente investigador en las escuela de pregrado en Universidad Cesar Vallejo Lima - Perú. E-mail: gautamavv@hotmail.com ORCID: https://orcid. org/0000-0001-7181-7497 
Barra Tello, Tamara Carolina; Salvatierra Melgar, Angel; Candia Haro, Isaac Moisés; Vargas-Vargas, Gautama

Gestión de riesgo de desastres en el marco de la cultura preventiva

hospital una vez producido el evento. Los colaboradores del hospital desconocen cómo actuar frente a un desastre natural por la falta de capacitación sobre las medidas de prevención de riesgo para mejorar las capacidades de respuesta ante eventos adversos.

Palabras clave: gestión de riesgo; desastres; conciencia; prevención

\title{
Disaster risk management within the framework of a preventive culture
}

\begin{abstract}
Facing the environmental problem that the planet is experiencing is one of the greatest challenges in guaranteeing the permanence of the human being, as expressed in the United Nations Scientific Conference, also known as the First Earth Summit; strategically planned and applied risk management paves the way to obtain a competitive advantage and a decisive advantage for public organizations. The objective of the research was to analyze disaster risk management in the macro of preventive culture from the lived experiences of workers in a public hospital in Lima. The present study is framed in the interpretive paradigm under a qualitative approach, as for the research design, Hernández \& Mendoza (2018) frame it within the interpretative phenomenological design when describing, exploring, but above all when understanding the context in which it is develops the investigation due to the flexibility of the process that is adapted to each reality, the analysis unit was 10 collaborators of the hospital brigade. The results indicate that the preventive culture has a positive impact on public hospital workers; impacting prevention positively not only in limiting the severity of the damage caused by the disaster, but also in the recovery capacity that the hospital will have once the event has occurred. Hospital employees do not know how to act in the face of a natural disaster due to the lack of training on risk prevention measures to improve response capacities to adverse events.
\end{abstract}

Keywords: risk management; disasters; awareness; prevention; planet; collaborators

\section{Introducción}

El medio ambiente en nuestro ecosistema, se encuentra en degradación. Es deplorable ver la situación de contaminación, la pérdida de la biodiversidad, el agotamiento de la capa de ozono, la desertificación y sequía; problemas que se han desencadenado por la actitud del hombre quien, en sus procesos de producción y reproducción social, construye un entorno que afecta cada día, no solo a la naturaleza, sino también a su mismo ser. Para poder darle el verdadero valor a nuestro medio ambiente y al cuidado del mismo, es urgente que se instaure una educación al respecto, que trabaje por 
generar y desarrollar en cada individuo una conciencia ambiental, y con esto se instituya en las personas nuevos hábitos de sentimiento de cuidado y protección hacia el medio en el que transcurre la vida (Morejón, 2006).

Al respecto, Latinoamérica registra una peculiar proclividad para la siniestralidad dadas las características sociales de sus poblaciones y las del entorno geográfico donde se asientan. El territorio muestra gran exposición a amenazas eminentemente sismotectónicas en el litoral del Pacífico, por ser una de las regiones más activas del Círculo de Fuego del Pacífico y predominantemente hidrometeorológicas en el litoral atlántico. Los terremotos acontecidos en las últimas dos décadas recuerdan, que América Latina se encuentra entre las zonas de alta vulnerabilidad a sismos y zonas con mucho hacinamiento ubicados en entornos complicados como cerros y laderas de ríos. Países como Brasil, Costa Rica, Cuba, México están entre los que han registrado el mayor progreso en la reducción de riesgos de desastres; demostrando de esta manera que cuentan con una gran cultura de gestión de riesgos y han tenido la oportunidad de superarlas como menciona Elahi (2013: 25). Es evidente, además de ser una cuestión desafiante, establecer, gestionar y evaluar la cultura de gestión de riesgos, como fuente de ventaja competitiva para las actuales instituciones públicas o privadas.

La justificación de forma teórica se sustenta en el estudio realizado por Narváez et al, (2009) respecto la gestión del riesgo de desastre (GRD), desde el enfoque por procesos, se argumentan en dos perspectivas fundamentales para encaminar los procesos: 1) la generación del riesgo y, 2) las diversas formas de intervenirlo. Desde la perspectiva práctica de la investigación, esta se plantea con beneficios a nivel de los colaboradores de un hospital público, adquiriendo información y/o conocimiento de esta herramienta de gestión social - ambiental, coadyuvando en su trajinar diario con el propósito de advertir y/o minimizar el riesgo ante cualquier fenómeno natural o antropológico.

Los centros de salud tienen una importancia respecto al enfrentamiento a estos desastres, en la experiencia clínica en los hospitales del Ministerio de Salud se evidencia cada vez más un incremento en la demanda de atenciones por emergencias, tal es así que en la última década, las emergencias en hospitales nacionales han ido en aumento producto de la actual pandemia que se presenta a partir del COVID-19.

Se analiza la gestión de riesgo de desastres en el macro de la cultura preventiva desde las experiencias vividas de los trabajadores de un hospital público ubicado en el Cercado de Lima, siendo un centro de atención estratégico, en caso de algún desastre, por lo que esta situación otorga relevancia a la investigación. El hospital seleccionado para el estudio, representa una unidad de análisis conformado por los trabajadores, quienes fungen como informantes clave en el proceso de recolección de información relativa a la gestión de riesgo del desastre, considerando las experiencias vividas en torno al tema investigado.

Enfrentar el problema ambiental que vive el planeta, es uno de los retos más grandes para garantizar la permanencia del ser humano. Según lo expresado en la Conferencia Científica de las Naciones Unidas también conocida como la Primera Cumbre 
para la Tierra, celebrada en Estocolmo (Suecia) en 1972, este problema sigue siendo un reto en la actualidad, si bien es cierto, existen varias formas de ser abordado, hay dos temas importantes que deben ser los baluartes que lleven las riendas de este problema: 1) la educación $y, 2$ ) la investigación. De lo dicho, se debe de tener en cuenta que las universidades son importantes y su papel es determinante en la solución de estos problemas en informar desde la formación profesional del estudiante unviersitario sobre la importancia del conocimiento de gestión del riesgo de desastre (Ochoa \& Hidalgo, 2016).

Desde la perspectiva metodológica, el presente estudio se enmarca en el enfoque cualitativo. Los aportes de Sánchez y Reyes (2015) determinaron que en un estudio cualitativo la información se procesa a través del análisis e interpretación de datos. En cuanto al tipo de investigación, Ayala (2008) menciona que está relacionado con la comprensión de la realidad. El método inductivo es el más apropiado para el presente estudio de acuerdo con lo propuesto debido al análisis de una realidad particular emitiendo un juicio general.

Asimismo, el diseño de la investigación es fenomenológica, Hernández \& Mendoza (2018), manifiestan que en la investigación cualitativa la realidad se determina mediante las interpretaciones de los participantes y del investigador en función de su misma realidad, generándose interacción de los actores a través de varios puntos de vista. Asimismo, se explora, describe y comprende experiencias de las personas respecto a un fenómeno. Por lo que el presente estudio tuvo como escenario de estudio el hospital público.
La unidad de análisis estuvo conformada por diez colaboradores brigadistas del hospital nacional de Lima, que pertenecen a las diferentes áreas de servicio. Los brigadistas están bajo la dirección del Centro de Operaciones de Emergencias (COE), conformados por médicos, enfermeras, técnicos, administrativos, seguridad, choferes y otros que se encuentran debidamente organizados, entrenados y equipados para estar en la capacidad de identificar las condiciones de riesgo que puedan generar determinadas emergencias $y$ así mismo se encuentran entrenados para actuar oportunamente controlando o minimizando sus consecuencias de dichos riesgos identificados.

\section{Gestión de riesgo de desastre}

\section{De acuerdo con Bozaykut-} Bük (2017:27) la gestión de riesgos estratégicamente planificada y aplicada allana el camino para obtener una ventaja competitiva y una ventaja decisiva para las instituciones financieras mundiales. La importancia de la gestión de riesgos se hace más evidente en los periodos de inestabilidad financiera. Para el autor, durante los últimos años, en el sector público de salud, existen falencias como se ha evidenciado en la actual pandemia.

Para Polo (2013:18), la definición de cultura ambiental se refiere a enseñar a prevenir y proteger el medio ambiente, con un enfoque en puntos importantes para conservar el recurso natural, proteger la fauna y flora, etcétera, en orientar en el entendimiento respecto a la relación que tiene el ser humano con su entorno con el fin de que se gestione adecuadamente los recursos naturales.

Asimismo, según la Comisión Nacional de Prevención de Riesgos y 
Atención de Emergencias (2014:16), se entiende por riesgo a la posibilidad de que se vaya a presentar alguna pérdida, daño, que puede ser económico, social o ambiental, en un entorno único durante un tiempo definido. Dicha Comisión señala que la gestión de riesgos representa una serie de procedimientos que buscan revertir de manera positiva las condiciones vulnerables de la una comunidad, y su entorno. Haciendo un modelo sostenible y de prevención, en el cual se va a incorporar factores efectivos de prevención y mitigación de desastres dentro de un plan territorial, sectorial y socioeconómico.

En los últimos años se han producido innumerables emergencias ocasionadas por fenómenos naturales, esto presupone que el mundo en que se está viviendo actualmente, es necesario conocer los problemas sanitarios con el propósito de reorganizarse para mitigar de forma correcta este tipo de eventos.

Al respecto, el Instituto Nacional de Defensa Civil (2010:57), alega que la gestión de riesgos establece una metodología para aplicar de manera sistemática, políticas, procedimientos y prácticas de identificación de tareas, análisis, evaluación, tratamiento y acciones para el monitoreo de riesgos.

Sobre este particular, la Secretaría Nacional de Gestión de Riesgos (SNGR) (2012), indica que estos procedimientos involucran tanto del sector público como al privado, por lo que deben asumirlos $y$ ejercerlos de manera correcta y adecuada, en sintonía con las normas establecidas. El fin es proteger a la comunidad de los desastres naturales, mitigándolos, y buscando recuperar y mejorar las condiciones del entorno desde el punto de vista social, económico y ambiental. De esa manera se trata de minimizar las condiciones vulnerables.

\section{Paradigmas de la gestión de riesgo del desastre}

Desde la posición de Ocampo y Santa (2019), a través de la historia se han producido desastres. El cambio se produce en la década de los '90 cuando aparece un enfoque distinto en el que sobresale el disponer de conocimientos sobre el riesgo.

Existen cuatro paradigmas no fijos en esta materia:

a) Gestión Prospectiva, en la que se toman las acciones para lograr la disminución del riesgo de desastre, por lo tanto, se busca impedir la generación de riesgos futuros, es decir estudiar el riesgo próximo e insertar mecanismos para manejar la inseguridad y la definición de contextos alternativos, en ese sentido se consideren los posibles cambios en las amenazas y la vulnerabilidad.

b) Gestión Correctiva, en este paradigma se busca la protección de medidas sujetadas a la organización del desarrollo que genere la disminución del riesgo de desastre actual, vale decir, la reducción de los riesgos existentes y sus factores que lo componen

c) Gestión Reactiva, en este modelo el riesgo de desastre no es absoluto, por lo que existirán riesgos excedentes que deben ser atendidos y que periódicamente se convertirán en desastres. Para ello se considera las medidas de reducción, toman acciones para hacer frente una vez que se desborde.

d) Gestión Compensatoria, en esta fase se busca la adopción y combinación de diferentes herramientas económicas, para hacer frente los posibles desastres.

\section{Procesos de la gestión del riesgo de desastres}


Barra Tello, Tamara Carolina; Salvatierra Melgar, Angel; Candia Haro, Isaac Moisés; Vargas-Vargas, Gautama

Gestión de riesgo de desastres en el marco de la cultura preventiva

Según el Instituto nacional de defensa civil - INDECI (2010) los procesos de la gestión del riesgo de desastres son:

- Estimación del riesgo, proceso que está constituido por acciones, actividades y procedimientos que se realizan para determinar la naturaleza y el grado de riesgo existente y futuro. Una evaluación en todo su conjunto de los riesgos no solo evalúa la magnitud y probabilidad de pérdidas potenciales en caso de un desastre, sino que también proporciona una comprensión completa de las causas y las consecuencias del impacto de esas pérdidas.

- Prevención y reducción del riesgo, proceso constituido por el conjunto de acciones, actividades y procedimientos que se orientan a minimizar las vulnerabilidades y riesgos existentes, prevenir la generación de futuros riesgos en la sociedad, y a la preparación de la respuesta ante emergencias y desastres.

- Preparación, respuesta y rehabilitación, proceso constituido por el conjunto de acciones y actividades, que se ejecutan durante un desastre o inmediatamente ocurrido éste, así como ante la inminencia del mismo, a fin de garantizar una adecuada y oportuna atención de las personas afectadas y damnificadas, así como la rehabilitación de los servicios básicos.

- Reconstrucción, constituido por el conjunto de acciones y actividades, que se desarrollan simultáneamente, para la recuperación humanitaria o social de damnificados y afectados, acciones de reactivación de las condiciones básicas de trabajo y del funcionamiento de los sistemas sociales y productivos.

- En esta misma línea se plantean los componentes de la gestión del riesgo de desastre, resaltando los siguientes aspectos según los autores Giannakis \& Papadopoulos (2016), Velásquez (2016), Calderón \& Frey (2017), Díaz, et al, (2018), Méndez, et al, (2011), Ávila, et al, (2017), Heras, \& Gavari (2019):

- Conocimiento del riesgo para Giannakis \& Papadopoulos (2016: 97), las fallas son inevitables y son parte de nuestras vidas, sin embargo, puede haber variaciones en la organización después de los errores, dependiendo del nivel. La preparación de una brigada puede hacer frente a un desastre $y$, en consonancia con los planes finalizados, se puede recuperar de ella. Por lo tanto, las actividades de preparación contribuyen a la capacidad de la brigada para evolucionar a problemas y son los primeros pasos que deben tomarse que le permiten convertir una tragedia en una oportunidad. La reducción de riesgos son movimientos y técnicas que se pueden realizar para generar experiencia en los riesgos o riesgos que están presentes, analizan lo vulnerable estableciendo niveles de riesgos en el contexto de la gestión del desarrollo sostenible, permitiendo que se tomen acciones respecto a la forma de cómo controlar el posible desastre.

Desarrollar capacidad de respuesta ante emergencias y desastres, para Velásquez (2016:78), es importante que se universalice los temas respecto a la salud, en donde toda la 
comunidad, tenga los beneficios con las acciones que realiza el Estado, con el fin de que se mejore los temas sociales, accediendo a mejores circunstancias a nivel individual y colectivo, sin el riesgo de llegar a niveles de pobreza como una consecuencia lógica ante los cuidados de la salud.

Fortalecer las capacidades institucionales para el desarrollo de la gestión del riesgo de desastres, para Calderón \& Frey (2017: 11), cuando en su estudio se refiere a institucionalizar la gestión de riesgo, esta cuenta con normas que son coordinadas de manera conjunta entre todos los actores involucrados. En este caso es el Estado quien debe regular los riesgos, debiendo asegurarse de que se construya una sociedad sobre la base de la elaboración de herramientas para que se deleguen funciones, en base a un organigrama estructurado entre diferentes sectores y territorios.

Fortalecer la participación de la población y sociedad organizada para el desarrollo de una cultura de prevención, para Sandoval, et al, (2018: 68) es importante que participe la comunidad, se empoderen y su resiliencia cobren una real importancia, buscando énfasis en enfocar sitios en que se reduzca los riesgos, a partir de trabajos humanitarios, con el mejoramiento de la capacidad sustentable para el desarrollo.

Cultura preventiva, de acuerdo con Méndez, et al, (2011) la cultura preventiva refiera al conjunto de ideas, valores, actitudes, modos de vida, expresados en patrones de comportamiento estables dentro de una sociedad, de modo que ayuden a guiar, ordenar, promover una conducta deseada y esperada entre sus miembros. Sin embargo está referida a la postura ontológica y axiológica sobre como la organización percibe el riesgo laboral, sus consecuencias y la forma de enfrentarlo. Incluye implantar políticas preventivas, su ejecución, evaluación e incluso modificación.

Protección del medio ambiente, para Ávila, et al, (2017:94), definieron a esta fase como los principios rectores de una adecuada política pública para contemplar el derecho humano al medioambiente. Esto plantea que se debe dar énfasis a los derechos de las personas y los principios que responsabilizan al Estado. Esto va a ayudar a que se comprenda el contenido y alcance de lo indicado. Principios sin derechos permiten mayor discrecionalidad por parte del Estado. Derechos sin principios disminuyen el peso del rol estatal en la protección de la naturaleza.

Educación ambiental, definida por Pulido \& Olivera (2018), como la educación para el desarrollo sostenible, es una tendencia que busca involucrar a estudiantes y profesores en los procedimientos del proceso de enseñanza-aprendizaje con el objetivo de que se genere conciencia sobre cómo preservar el medio ambiente.

Capacidad de resiliencia, para Pastrana, Potenciano de la Heras \& Gavari (2019), se refiere la capacidad de las personas que viven en una comunidad de hacerle frente a algún desastre, en el cual se condiciona por la fortaleza o debilidad que se presente. Estas fortalezas y debilidades se condicionan por este concepto, que más que todo es como se adaptan las personas al momento de que se dé una situación adversa. 


\section{Gestión de riesgos en hospitales: Resultados y discusión}

Los resultados obtenidos están en función de las respuestas que dieron los colaboradores, de acuerdo con esto, es necesario indicarles que, en función de lo que indican los participantes, se tiene los siguientes resultados:

La cultura preventiva incide de forma positiva en los trabajadores del hospital público, así como para hacerle tomar conciencia sobre los trabajadores. La prevención impactará positivamente no solo en la limitación de la gravedad de los daños que produzca el desastre, sino también en la capacidad de recuperación que tendrá el hospital una vez producido el evento. Ayudará a prevenir accidentes laborales, identificar oportunamente patologías y tratarlas, maximizando la participación de los trabajadores en las actividades destinadas a la preparación y respuesta ante desastres, logrando así una mayor resiliencia de la institución. Al respecto la Organización Internacional del Trabajo (OIT), indicó que es un punto clave para la gestión de la seguridad y la salud promover una cultura de prevención dentro de la empresa. Sin embargo, la disminución de las lesiones $y$ enfermedades profesionales ha sido mínima, dando lugar a un alto interés en la gestión de la salud y la seguridad de sistemas.

La gestión de la información estandarizada se desarrolla a partir de formar capacitaciones que se puedan extender a todas las áreas del hospital , además de que sea una información válida y acertada respecto a lo que se trate, también desarrollar parámetros claros y objetivos de tal manera que la información sea útil, relevante, veraz y oportuna, mediante el uso de herramientas validadas con es el Sireed o la información que maneja el EMED, así como el manejo de información ante la activación del sistema de comando de incidentes hospitalario.

Para Velásquez (2016: 78), es importante que se universalicen los temas respecto a la salud, en donde toda la comunidad, tenga los beneficios con las acciones que realiza el Estado, con el fin de que se mejore los temas sociales, accediendo a mejores circunstancias a nivel individual y colectivo, sin el riesgo de llegar aniveles de pobreza como una consecuencia lógica ante los cuidados de la salud.

Los entrevistaron manifestaron que el proceso de planificación del ordenamiento y gestión de riesgo se debe fortalece proyectando las medidas preventivas a la práctica de acuerdo con la zona donde se ubiquen, mediante planos catastróficos, señalizaciones y buena creación de parques, saneamientos básicos, también con políticas territoriales dirigidas a disminuir los daños y la vulnerabilidad y ello no se aplica en el hospital. Este uso y aplicación de la normativa vigente reduce estas vulnerabilidades, riesgos y amenazas, creando equipos multidisciplinarios que deberían crearse en el hospital. Teniendo una política central que promueva la regulación y promoción de los trabajadores.

Los resultados coinciden con el estudio de Sandoval Díaz et al, (2018: $68)$, en el que manifiestan la importancia en la participación de los trabajadores de toda la organización; su empoderamiento y resiliencia, de manera que esto cobre una real importancia, buscando énfasis en enfocar sitios en los que se reduzca los riesgos, a partir de trabajos humanitarios, con el mejoramiento de la 
capacidad sustentable para el desarrollo. Existe un desconocimiento por parte de los trabajadores sobre las condiciones de seguridad de los servicios básicos y medios de vida, siendo estos esenciales ante el riesgo de algunos desastres se pueden desarrollar comenzando por examinar las zonas con mayor posibilidad de riesgo para encontrar soluciones factibles en cada una de ellas, pero para ello, se debe conocer las zonas de seguridad del hospital para ante un desastre poder refugiarnos.

Al respecto, Van der Merwe (2020) establece que la gestión del riesgo de desastres es un proceso multidisciplinario y multisectorial continuo e integrado de planificación e implementación de medidas que establecen una cultura de evitación de riesgos mediante la construcción de comunidades resilientes. Se debe desarrollar un plan de gestión de desastres integral investigado, pero solo tendrá éxito si establece prioridades claras; se practica, prueba, adapta y / o modifica cuando sea necesario; y es práctico, factible y bien entendido por todos los involucrados.

La capacidad de respuesta inmediata ante emergencias y desastres se desarrolla a partir de charlas, capacitaciones y práctica constante de lo aprendido, entre grupos de intervención rápida, apoyo logístico. Mediante la capacitación de brigadas especializadas en primera intervención ante emergencia y desastres, quienes trabajaran estrechamente con el área de emergencia y cuidados críticos; asumiendo la adecuada implementación de las mencionadas brigadas.

La capacidad para la atención de emergencias y desastres se desarrolla mediante planes de atención y planes de contingencia, así como a través de la coordinación, la promoción y el impulso de las acciones de gestión de riesgo. Mediante la identificación oportuna de las vulnerabilidades, riesgos $y$ amenazas de la institución, trabajando planes de acción y contingencia para múltiples escenarios y riesgos, llevando a cabo ejercicios de preparación a fin de fortalecer la capacidad de respuestas.

Las condiciones para una gestión efectiva en la rehabilitación y reconstrucción después de un desastre se implementan realizando una lista de daños, analizando la gravedad de estos para posteriormente atenderlos acorde la situación del asunto, mediante un buen diagnóstico de la magnitud de un desastre y principalmente con la designación de inversiones del estado. Trabajando de manera articulada con los tres niveles de gobierno teniendo como ente articulador a Indeci y a Digerd, priorizando las necesidades más importantes a ese momento.

La institucionalidad de la gestión de riesgos en los tres niveles de gobierno se da a través de estrategias impuestas por la defensa nacional utilizando recursos gerenciales con una adecuada articulación en las plataformas de gestión de riesgo. También se puede dar efectivizando la normativa dada por PCM donde se indica la obligatoriedad de la aplicación de la GRD en los diferentes niveles de gobierno, así como en las instituciones del Estado, así con la integración dentro de las estructuras orgánicas y funcionales de las instituciones.

Asimismo, los resultados obtenidos coinciden con el estudio de Calderón \& Frey (2017: 11), quienes refieren al institucionalizar la gestión de riesgo, es necesario que esta cuenta con normas que son coordinadas de manera conjunta entre todos los actores involucrados. 
Barra Tello, Tamara Carolina; Salvatierra Melgar, Angel; Candia Haro, Isaac Moisés; Vargas-Vargas, Gautama

Gestión de riesgo de desastres en el marco de la cultura preventiva

En este caso, es el Estado quien debe regular los riesgos asegurándose de que se construya una sociedad sobre la base de la elaboración de herramientas para que se deleguen funciones basado en un organigrama estructurado entre diferentes sectores y territorios.

La gestión de continuidad operativa se desarrolla estableciendo acciones para hacer frente a la ocurrencia de un evento inesperado con el objetivo de evitar la paralización de las operaciones. La continuidad operativa se debe evaluar de acuerdo con la emergencia a enfrentar, donde se debe activar el plan de contingencia ante determinado evento, desplegando los recursos necesarios priorizando las áreas principales de la institución. Se identifican los procesos claves sin los cuales no funciona la institución, luego de eso se asigna personal y equipamiento, así como personal y equipamientos alternos para cumplir la función.

Las capacidades que gestionen la protección del medio ambiente se desarrollan clasificando los residuos contaminados, para que de esta forma se proceda a su eliminación de una manera no dañina para el medio ambiente $y$ la salud. El Ministerio del Ambiente juntamente con todos los sectores del Estado tiene un presupuesto para designar para protección del ambiente. Teniendo en cuenta conceptos y acciones eco amigables y sostenibles en el desarrollo de las diferentes actividades a desarrollar en GRD. Al respecto, Cardone (2017: 67) indica que la labor científica lejos de estar desconectada de la labor social invita a la responsabilidad de los distintos sujetos que viven en el planeta, en su visión más heterogénea, a ser parte consciente del proceso de producción de los desastres en sí mismos, cómo en el proceso que se gesta en torno al riesgo con el objetivo de disminuirlo.

La cultura de prevención y restablecimiento frente a amenazas se desarrolla por medio de charlas, capacitaciones y comunicación horizontal constante, fortaleciendo las actividades preventivas promocionales. Tratando de generar conciencia y responsabilidad en el personal promoviendo una cultura de prevención ante evento multiamenazas, motivándolos y capacitándolos para desarrollar acciones determinadas ante la ocurrencia de algún evento.

Analizar el fortalecimiento de la participación de la población y sociedad organizada para el desarrollo de la cultura de prevención de los trabajadores del Hospital Nacional, Lima. La gestión de riesgo de desastre se fortalece a partir de charlas, capacitaciones, y con constante información acerca de las medidas de prevención de riesgo a todo el personal creando una cultura de prevención y mejorando las capacidades de respuesta ante eventos adversos teniendo en cuenta las nuevas políticas de trabajo de gestión de riesgos y desastres. La cooperación entre naciones, es independiente a la gestión de riesgos, las naciones tienen formas diferentes de conceptualizar los términos (Velásquez, 2016).

\section{Conclusiones}

La gestión de riesgo de desastres (GRD) se cultiva cada vez más y su práctica, está siendo reconocida como intensa debido a que las ciencias de la administración vienen profundizándose cada vez más, los cual está dando buenos resultados, es necesario que tanto los trabajadores de los hospitales públicos, como la institución que contribuirá, adopten medidas para 
prevenir y/o minimizar riesgos ante futuros fenómenos naturales. Los conocimientos logrados están siendo utilizados para generar un sistema de reducción de riesgo de desastres, que aprovecha la solidez de las capacidades institucionales, que es un asunto que está ocurriendo por el progreso de la ciencia, la tecnología, la educación, la salud y la cultura de los actores claves de la vida social pero también de las instituciones existentes en el gobierno, el sector privado y la sociedad civil.

Los colaboradores del hospital desconocen cómo actuar frente a un desastre natural por la falta de capacitación sobre las medidas de prevención de riesgo para mejorar las capacidades de respuesta ante eventos adversos. No se cuenta con un adecuado proceso de planificación del ordenamiento y gestión territorial para fortalecer las medidas preventivas a la práctica de acuerdo con la zona donde se ubiquen, y afrontar adecuadamente ante futuros fenómenos naturales a los cuales son vulnerables. Los colaboradores deben conocer su función específica en el plan y estar debidamente capacitado para ejecutarlo. Fortalecimiento de capacidades en la administración de desastres es también un proceso multisectorial, multidisciplinario e integrador basado en programas educativos integrados, de entrenar y de conciencia pública en toda organización sanitaria.

\section{Referencias bibliografica}

Ávila, R., Campusano, R., Galdámez, L., Jariai, J., \& Lucas, A. (2017). Medioambiente, conflictos socioambientales y derechos humanos. Anuario de Derechos Humanos, (13), 13-39. https://doi. org/10.5354/0718-2279.2017.46883

Ayala, R. (2008). La metodología fenomenológico-hermenéutica de M. Van Manen en el campo de la investigación educativa. Posibilidades y primeras experiencias. Revista de Investigación Educativa, 26(2), 409430. https://revistas.um.es/rie/article/ view/94001

Bozaykut-Bük, T. (2017). Giving Risk Management Culture a Role in Strategic Planning. En H. Dinçer, \& Ü. Hacioğlu (Edits.), Risk Management, Strategic Thinking and Leadership in the Financial Services Industry (págs. 311-321). Estambul: Springer. https://doi.org/10.1007/978-3-31947172-3 21

Calderón, D. R., \& Frey, K. (2017). El ordenamiento territorial para la gestión del riesgo de desastres en Colombia. Territorios, (36), 239-264. http://dx.doi.org/10.12804/revistas. urosario.edu.co/territorios/a.4795

Cardone, V. (2017). Estudio sobre la gestión del riesgo en desastres ambientales naturales. https://bit. Iy/2QRHzBW

Comisión Nacional de Prevención de Riesgos y Atención de Emergencias. (2014). Normas y elementos básicos de gestión municipal del riesgo de desastre con énfasis en prevención, control y regulación territorial. San José, Costa Rica: La Comisión, Sistemas Geoespaciales. https://bit. Iy/3tO2V1C

Elahi, E. (2013). Risk management: the next source of competitive advantage. Foresight, 15(2), 117-131. https://doi. org/10.1108/14636681311321121

Giannakis, M., \& Papadopoulos, T. (enero de 2016). Supply chain sustainability: A risk management approach. International Journal of Production Economics, 171(4), 455-470. https:// 
Barra Tello, Tamara Carolina; Salvatierra Melgar, Angel; Candia Haro, Isaac Moisés; Vargas-Vargas, Gautama

Gestión de riesgo de desastres en el marco de la cultura preventiva

\section{doi.org/10.1016/j.ijpe.2015.06.032}

Hernández, R., \& Mendoza, C. (2018). Metodología de la investigación: las rutas cuantitativa, cualitativa y mixta. McGraw-Hill Education.

Instituto Nacional de Defensa Civil del Perú. (2010). Atlas de peligros del Perú 2010. Lima: Indeci.

Méndez, E., Figueredo, C., Chirnios, E., Goyo, A., \& Rivero, E. (2011). Cultura preventiva del riesgo laboral del docente universitario. Revista Multiciencias, 44-49. https://www. redalyc.org/pdf/904/90418851006. pdf

Morejón, A. (1 de octubre de 2006). Formación de la conciencia ambiental, importancia de la ética ambiental y la educación ambiental en este proceso. https://bit.ly/3dMirFu

Narváez, L., Lavell, A., \& Pérez Ortega, G. (2009). La gestión del riesgo de desastres: un enfoque basado en procesos. Lima: Secretaría General de la Comunidad Andina.

Ocampo, M., y Santa Catarina, C. (2019). Gestión del Riesgo de Desastres. INCYTU, (33), 1-6.

Ochoa Henríquez, O. J., \& Hidalgo López, C. R. (2016). Caracterización de la cultura ambiental en la gestión de la Universidad Centroccidental Lisandro Alvarado. Compendium, 19(37), 27-53. https://www.redalyc. org/pdf/880/88049677003.pdf

Pastrana, J., Potenciano de la Heras, Á., \& Gavari, E. (2019). Gestión de riesgo de desastres y protección civil en España: aportes para el desarrollo de una cultura preventiva. Revista de Estudios Latinoamericanos sobre Reducción del Riesgo de Desastres Reder, 3, 44-57. http://revistareder. com/ojs/index.php/reder/article/ view/31/33
Polo, J. C. (octubre-diciembre de 2013). El Estado y la educación Ambiental Comunitaria en el Perú. Acta Médica Peruana, 30(4), 141-147. http:// www.scielo.org.pe/pdf/amp/v30n4/ a17v30n4.pdf

Pulido, V., \& Olivera, E. (2018). Aportes pedagógicos a la educación ambiental: una perspectiva teórica. Revista de Investigaciones Altoandinas, 20(3), 333-346. http:// dx.doi.org/10.18271/ria.2018.397

Sánchez, H. y Reyes C. (2015). Metodología y Diseños en la Investigación Científica. Editorial Business Support Aneth S.R.L

Sandoval Díaz, J. S., Rojas Páez, L., Villalobos Soublet, M., Sandoval Díaz, C., Moraga R., F., \& Aguirre P., N. (mayo de 2018). De organización vecinal hacia la gestión local del riesgo: diagnóstico de vulnerabilidad y capacidad. Revista INVI, 33(92), 155-180. http://dx.doi.org/10.4067/ S0718-83582018000100155

Secretaría Nacional de Gestión de Riesgos. (2012). Manual del Comité de Gestión de Riesgos. https:// reliefweb.int/sites/reliefweb.int/files/ resources/Informe completo 20.pdf

Van der Merwe, P. (2020) Prevention and preparedness: biosecurity, early warning and contingency planning. Rev. Sci. Tech. Off. Int. Epiz., 39 (2), 551-559 http://dx.doi:10.20506/ rst.39.2.3106

Velásquez, A. (julio-septiembre de 2016). Salud en el Perú: hacia la cobertura universal y una respuesta efectiva frente a riesgos sanitarios. Revista Peruana de Medicina Experimental y Salud Publica, 33(3), 397-398. http://dx.doi.org/10.17843/ rpmesp.2016.333.2339 